\title{
DVCS measurements at HERMES
}

\author{
Klaus Rith* (on behalf of the HERMES collaboration) \\ Friedrich-Alexander-Universität Erlangen-Nürnberg \\ E-mail: klaus.rith@physik.uni-erlangen.de
}

\begin{abstract}
Deeply Virtual Compton Scattering (DVCS) is currently one of the most promising processes that provides information about the structure of the nucleon in the framework of Generalized Parton Distributions (GPDs). During its last years the deeply virtual Compton scattering process was extensively studied at the HERMES experiment through measurements of cross-section asymmetries. To the virtue of unique experimental conditions, HERMES had collected a wealth of data on scattering a longitudinally polarized lepton (electron/positron) beam off unpolarized, longitudinally and transversely polarized hydrogen targets, as well as off unpolarized and longitudinally polarized deuterium targets. During the last two years of HERA operation a recoil detector around the target area allowed for the full kinematic reconstruction of the exclusive DVCS event, leading to a clean measurement of the beam-helicity asymmetry with background well below the $1 \%$ level. The recoil detector also allowed for first measurement of the related process of associated DVCS, in which the proton is excited to a Delta resonance, giving access to transition GPDs. In this contribution these recent results using the recoil detector are presented.
\end{abstract}

XXII. International Workshop on Deep-Inelastic Scattering and Related Subjects, 28 April - 2 May 2014

Warsaw, Poland

\footnotetext{
* Speaker.
} 


\section{Introduction}

An important part of the experimental program of the HERMES experiment at HERA [1] has been the measurement of azimuthal asymmetries in Deeply Virtual Compton Scattering (DVCS), i.e., the hard exclusive electroproduction of real photons, $e N \rightarrow e^{\prime} N \gamma$. The DVCS process involves two hard vertices, the interaction of the virtual photon with a quark and the radiation of a real photon by the struck quark, whereby the target nucleon $\mathrm{N}$ is left intact. DVCS competes with the BetheHeitler $(\mathrm{BH})$ process, where a real photon is radiated by the initial or final state lepton. These two processes have identical final states and their amplitudes interfere. The contributions of the DVCS, BH and interference (I) term to the cross section can be disentangled by the measurement of azimuthal cross-section modulations. The relevant azimuthal angles are the angles $\phi$ and $\phi_{s}$ around the direction of the exchanged virtual photon between the lepton scattering plane and the production plane of the real photon and the (transverse) target spin direction, respectively. The amplitudes of these modulations are usually denoted as $A_{X Y}^{F . H}$. Here, the first subscript refers either to the beam charge $(\mathrm{C})$ or to the beam polarisation (U: unpolarised, L: longitudinally polarised), the second subscript refers to the target polarisation (U, L, T: transversely polarised), and $F . H$. denotes Fourier harmonics of linear combinations of $\phi$ and $\phi_{s}$.

Such measurements allow to constrain Generalised Parton Distributions (GPDs) which provide a framework for describing the multidimensional structure of the nucleon (for a review see, e.g. Ref. [2]). These GPDs encompass parton distribution functions and elastic nucleon form factors as limiting cases and provide correlated information on transverse spatial and longitudinal momentum distributions of partons. They depend on the kinematic variables $t, \xi, x$ and $Q^{2}$. Here, $t=\left(P-P^{\prime}\right)^{2}$ is the squared difference between the initial $(P)$ and final $\left(P^{\prime}\right)$ four-momenta of the target nucleon and $x(2 \xi)$ is the average (difference) of the longitudinal momentum fractions of the quark in the initial and final state. In hard exclusive leptoproduction, $x$ has no direct relationship with the experimental kinematic observable $x_{B}=Q^{2} /(2 P \cdot q)$, where $q$ is the difference between the fourmomenta of the incident and scattered lepton and $Q^{2} \equiv-q^{2}$. The parameter $\xi$ is related to $x_{B}$ by $\xi \approx x_{B} /\left(2-x_{B}\right)$. At leading twist, there are four chiral-even quark GDPs: $H, E, \widetilde{H}$ and $\widetilde{E}$. The GPDs $H$ and $\widetilde{H}$ conserve nucleon helicity, while $E$ and $\widetilde{E}$ are associated with a helicity-flip of the nucleon. DVCS is sensitive to all four distributions. The distributions $H$ and $E$ are of special interest since they may provide access to the total quark angular-momentum contribution $J^{q}$ to the nucleon spin through the Ji relation [3].

\section{Overview of DVCS measurement by HERMES}

The HERMES experiment is rather unique since it has performed such measurements using longitudinally polarised electron and positron beams with both helicities, with unpolarised and longitudinally polarised hydrogen $(\mathrm{H})$ and deuterium (D) targets, a transversely polarised $\mathrm{H}$ target and unpolarised nuclear targets. These various experimental conditions allowed to measure a large number of azimuthal modulations as a function of $t, x_{B}$ and $Q^{2}$.

An overview of the most important extracted asymmetry amplitudes integrated over the entire HERMES kinematic range is shown in the left panel of Fig. 1. Circles (triangles) denote data taken with $\mathrm{H}$ (D) targets and the standard detector configuration without recoil detector. The squares 

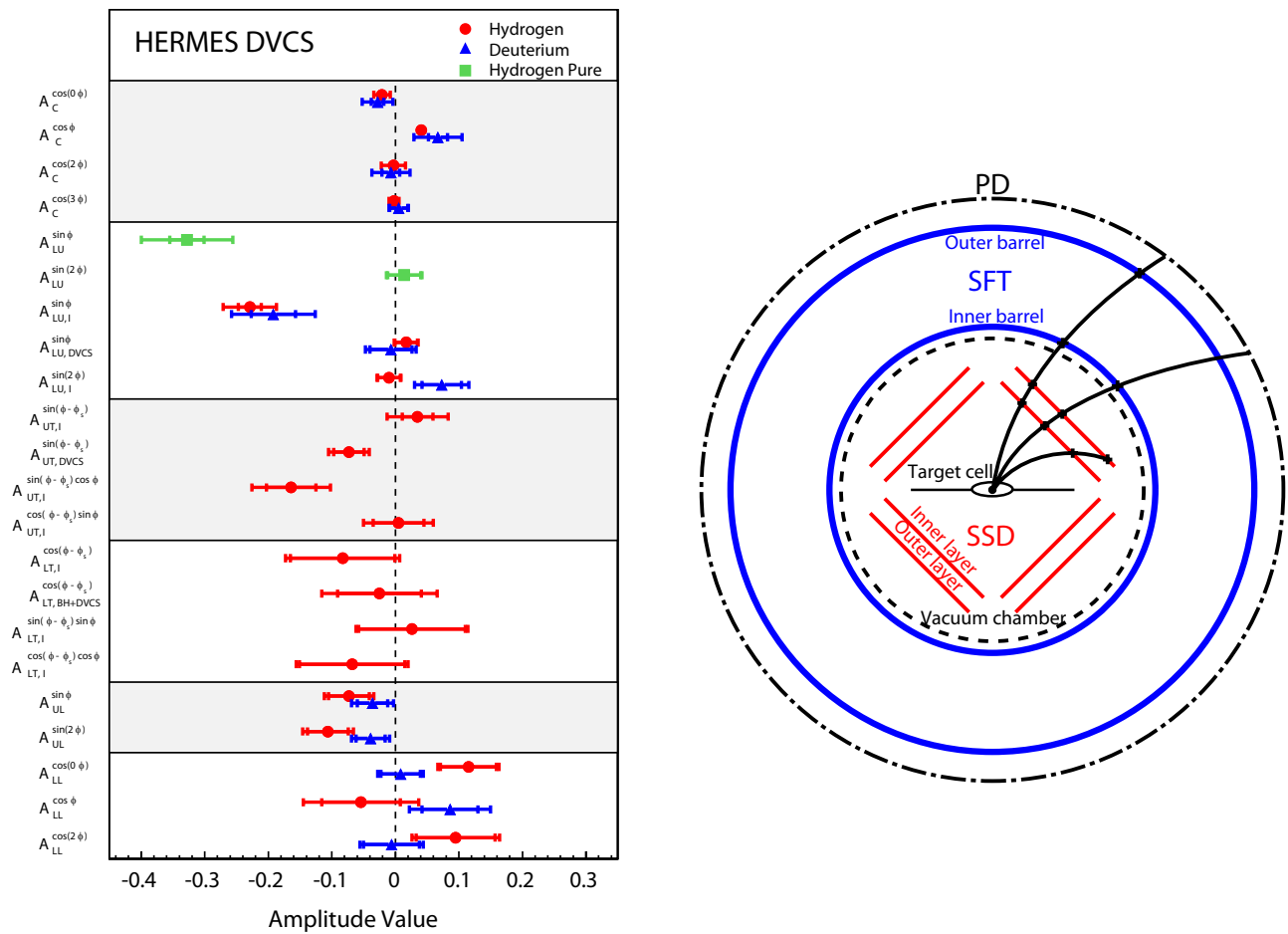

Figure 1: Various DVCS amplitudes integrated over the entire HERMES kinematic range (left); schematic diagram of the Hermes recoil detector (right).

denote data taken in 2006-2007 [4], where the recoiling proton was detected with the recoil detector [5], shown in the right panel of Fig. 1. In general, the amplitudes for $\mathrm{H}$ and $\mathrm{D}$ are very similar. In the upper two panels various amplitudes for beam-charge and beam-helicity asymmetries $[6,7]$ are shown. The amplitudes $A_{C}^{\cos (0 \phi)}$ and $A_{C}^{\cos \phi}$ are significantly different from zero. They are sensitive to the real part of the Compton form factor (CFF) $\mathscr{H}$. (CFFs are convolutions of GPDs with hard scattering kernels). Measurements with both beam charges and both beam helicities allow to disentangle the two components of the beam-helicity amplitude $A_{L U}^{\sin \phi}$ arising from the interference term and from the DVCS term. The latter is negligible compared to the former. The substantial beam-helicity amplitude $A_{L U, I}^{\sin \phi}$ is of special interest since it is sensitive to the imaginary part of $\mathscr{H}$. The transverse target-spin asymmetries [8] in the middle panels are sensitive to the GPD E. Here, the amplitude $A_{U T, I}^{\sin \left(\phi-\phi_{s}\right) \cos \phi}$ which is proportional to the imaginary part of a linear combination of the CFFs $\mathscr{H}$ and $\mathscr{E}$ is significantly different from zero. The amplitudes of the longitudinal target-spin asymmetries [9] are shown in the lower two panels. The $A_{U L}$ amplitudes in the second panel from below are sensitive to $\mathfrak{I} m(\widetilde{\mathscr{H}})$ and the longitudinal double-spin asymmetries $A_{L L}$ in the bottom panel to $\Re e(\widetilde{\mathscr{H}})$. Note that not all measured amplitudes are shown in this plot, but only a selection of the most important ones. For example, only four out the twelve (eight) extracted $A_{U T}$ $\left(A_{L T}\right)$ amplitudes [9] are presented. Also not shown are the amplitudes extracted from measurements with nuclear targets [10]. All these amplitudes are an important input for the extraction of GPDs from hard exclusive processes.

As an example, the kinematical dependences of the beam-charge asymmetry amplitudes (upper 


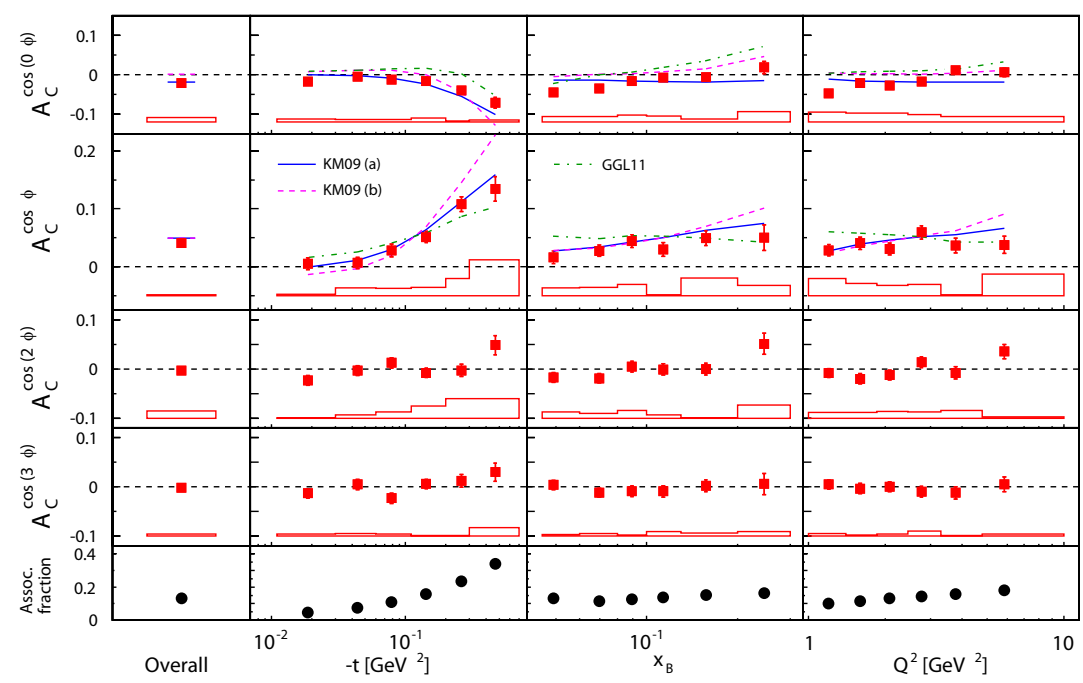

Figure 2: The beam-charge asymmetry amplitudes extracted from all unpolarised hydrogen data [7]. The error bars (bands) represent the statistical (systematic) uncertainties. Theoretical calculations are shown as solid and dashed lines (KM09) [11] or as dashed-dotted lines (GGL11) [12]. Also shown is the integrated value presenting the data in a single bin ('Overall'). The simulated fractional contribution from associated production to the event sample is shown in the bottom panel.

four entries in the left panel of Fig. 1) extracted from all unpolarised hydrogen data recorded at HERMES from 1996 until 2007 [7] are presented in Fig. 2. These amplitudes depend on the real part of combinations of the CFFs $\mathscr{H}, \mathscr{E}$ and $\widetilde{\mathscr{H}}$ that relate respectively to the GPDs $H, E$ and $\widetilde{H}$. At HERMES kinematics the contributions of $\mathscr{E}$ and $\widetilde{\mathscr{H}}$ are kinematically suppressed by more than an order of magnitude with respect to $\mathscr{H}$. Hence, GPD H can be constrained by such measurements. The amplitudes $A_{C}^{\cos 0 \phi}$ and $A_{C}^{\cos \phi}$ are proportional to the same combination of CFFs but with opposite sign as it is clearly seen in the t-dependence of these amplitudes. The amplitude $A_{C}^{\cos 0 \phi}$ is of twist- 3 and kinematically suppressed. The amplitude $A_{C}^{\cos 3 \phi}$ is of twist- 2 , but it is related to gluon helicity-flip GPDs and thus suppressed by $\alpha_{\mathrm{s}} / \pi$, where $\alpha_{\mathrm{s}}$ is the strong-coupling constant. At HERMES, the variables $x_{B}$ and $Q^{2}$ are strongly correlated. Therefore, the dependences of the amplitudes on these two variables are very similar.

For these measurements, the events were selected of having exactly one lepton track and no other charged-particle track and exactly one signal cluster with an energy deposit above $5 \mathrm{GeV}$ in the electromagnetic calorimeter not associated with the lepton track. The event sample was further selected requiring that the events fulfilled the kinematic constraints $1 \mathrm{GeV}^{2}<Q^{2}<10 \mathrm{GeV}^{2}$, $0.03<x_{B}<0.35,-t<0.7 \mathrm{GeV}^{2}, W>3 \mathrm{GeV}$ and $v<22 \mathrm{GeV}$, where $W$ is the invariant mass of the virtual-photon nucleon system and $v$ is the energy of the virtual photon in the target rest frame. Furthermore it was required that the squared missing mass $M_{X}^{2}=\left(q+M_{p}-q^{\prime}\right)^{2}$ corresponded to the squared proton mass within an 'exclusive region', $(-1.5 \mathrm{GeV})^{2}<M_{X}^{2}<(1.57 \mathrm{GeV})^{2}$. Here, $q^{\prime}$ is the four-momentum of the produced real photon. The data sample in this region does not only contain events in which the proton remains intact, but also events involving the excitation of the target proton to a $\Delta^{+}$resonant state ('associated production'). The momentum resolution of the spectrometer for the electron track and the energy resolution of the calorimeter for the real photon 
does not allow the separation of such events from the rest of the data sample. They are treated as part of the data. No systematic uncertainty is assigned for the contribution of these events. The fractional contribution to the event sample is shown in the last row of Fig. 2. It was estimated from a Monte Carlo simulation, the average contribution being about $12 \%$.

\section{Measurements with the Recoil Detector}

During the last two years of HERA operation, the HERMES spectrometer was extended by a recoil detector [5] which surrounded the target cell. The main purpose of this recoil detector was to record all particles in the final state of the DVCS process including the recoiling proton and hence to remove the events from associated production from the event sample. At HERMES energies, the majority of the recoiling protons in the DVCS process have momenta between $50 \mathrm{MeV}$ and $600 \mathrm{MeV}$ and polar angles in the range $0.5-1.35 \mathrm{rad}$. The recoil detector was designed to cover the full polar-angle range and most of the momentum range down to a lower limit of $125 \mathrm{MeV}$ which was imposed by the energy loss of the protons in the target-cell walls and the first detector layer. It consisted of three active detector parts: two layers of double-sided Silicon Strip Detectors (SSD) around the target cell inside the HERA beam vacuum, two layers of a Scintillating Fiber Tracker (SFT) and three layers of a Photon Detector (PD). They were surrounded by a magnet providing a longitudinal field. A cross-sectional view of the detector is shown in the right panel of Fig. 1. Also shown are examples of tracks reconstructed from two, three, and four space-points which are indicated by crosses. The energy deposited along the particle's track through the SSD and SFT was used to identify pions and protons and also to improve the accuracy of the momentum and angular resolution of the reconstructed tracks. For example, the momentum resolution of the proton reconstruction at a proton momentum of $150 \mathrm{MeV}$ is $\sigma\left(P^{\prime}\right) \approx 3 \mathrm{MeV}$, a really tiny number compared to momenta of several $\mathrm{GeV}$ of the particles recorded by the forward spectrometer.

A 'pure' exclusive data sample was selected by combining information from the forward spectrometer and the recoil detector in a kinematic event fit based on four-momentum conservation under the hypothesis of the process $e p \rightarrow e p \gamma$. Events were selected with high efficiency (83\%) and very small background contamination of less than $0.2 \%$. Results for the single-charge beamhelicity for this background-free 'pure' sample are shown in the left panel of Fig. 3 (circles). Also shown are the previously published results (asterixes) obtained by the missing-mass method without using recoil-detector information and results for an 'unresolved-reference' sample (triangles) requiring the hypothetical recoil proton to be in the acceptance of the recoil detector [4]. The magnitude of the overall $A_{L U}^{s i n} \phi$ amplitude for the pure sample is about $30 \%$ larger than the one for the unresolved sample. Consequently, the amplitude for the associated background must be very small, such that it can be considered as a dilution. Remarkably, the amplitudes for the pure sample agree much better with predictions made for this quantity based on data for hard exclusive meson production [13] than those for the unresolved sample.

In a further analysis the asymmetry for the associated fraction was determined separately under the assumption that it stems mainly from the reactions $e p \rightarrow e \gamma \pi^{0} p$ or $e p \rightarrow e \gamma \pi^{+} n$ in the $\Delta$ resonance region [14]. The amplitudes for associated DVCS contain interesting information since they give acces to transition GPDs. Kinematic event fitting was performed under the corresponding hypotheses, considering all recoil tracks identified as protons and positively charged pions. The 

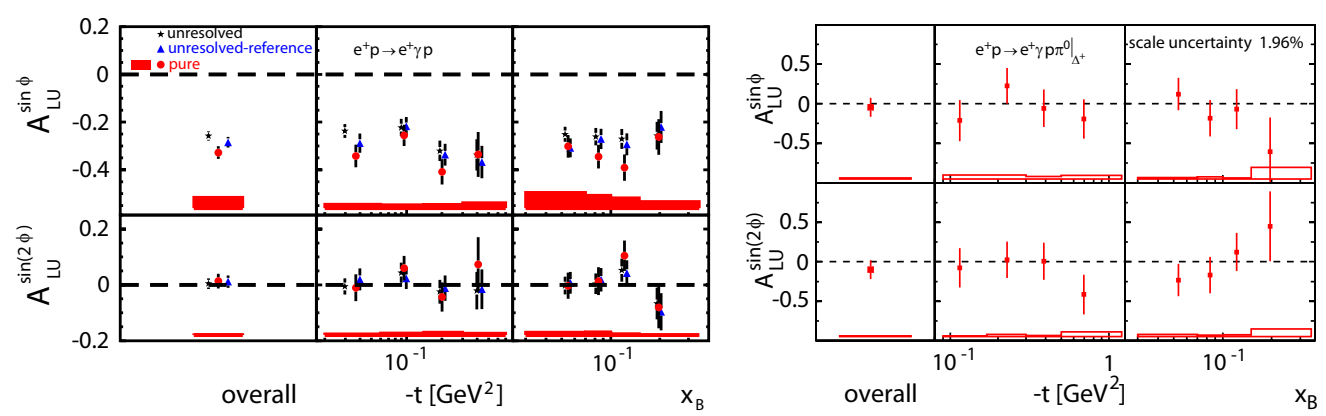

Figure 3: Amplitudes of the single-charge beam-helicity asymmetry obtained with recoil-proton reconstruction for the pure channel $e p \rightarrow e \gamma p$ (left) and for the associated channel $e p \rightarrow e \gamma \pi^{0} p$ (right). The 'overall' results shown in the very left panels are extracted in a single kinematic bin covering the entire kinematic acceptance. Statistical (systematic) uncertainties are represented by error bars (bands).

amplitudes for the channel $e p \rightarrow e \gamma \pi^{0} p$ with recoil-proton reconstruction are shown in the right panel of Fig. 3. Indeed, the 'overall' $A_{L U}^{\sin \phi}$ amplitude for this channel is consistent with zero. This is also the case for the channel with recoil-pion reconstruction. Presently, it seems to be a reasonable approach to assume that the associated background can be treated just as a dilution also for all the other DVCS amplitudes measured by HERMES and to correct the published asymmetry amplitudes accordingly. However, the differences between the old and the corrected values should then be included in their systematic uncertainties.

\section{References}

[1] K. Ackerstaff et al., Nucl. Inst. Meth. A 417 (1998) 230 [hep-ex/9806008].

[2] D. Müller et al., Fortschr. Phys. 42 (1994) 101 [hep-ph/9812448].

[3] X. Ji, Phys. Rev. Lett. 78 (1997) 610 [hep-ph/9603249]; Phys. Rev. D 55 (1997) 7114 [hep-ph/9609381].

[4] A. Airapetian et al., JHEP 1012 (2012) 042 [1206.5683].

[5] A. Airapetian et al., JINST 8 (2013) P05012 [1302.6092].

[6] A. Airapetian et al., Phys. Rev. Lett. 87 (2001) 182001 [hep-ex/0106068]; Phys. Rev. D 75 (2007) 011103 [hep-ex/0605108] ; JHEP 1109 (2009) 083 [0909.3587]; Nucl. Phys. B 829 (2010) 1 [0911.0095].

[7] A. Airapetian et al., JHEP 0712 (2012) 032 [1203.6287].

[8] A. Airapetian et al., JHEP 0608 (2008) 066 [0802.2499]; Phys. Lett. B 704 (2011) 15 [1106.2990].

[9] A. Airapetian et al., JHEP 0610 (2010) 019 [1004.0177]; Nucl. Phys. B 842 (2011) 265 [1008.3996].

[10] A. Airapetian et al., Phys. Rev. C 81 (2010) 0352002 [0911.0091].

[11] K. Kumerički and D. Müller, Nucl. Phys. B841 (2010) 1 [0904.0458].

[12] G. Goldstein, J. Hernandez and S. Liuti, Phys. Rev. D 84 (2011) 034007 [1012.3776].

[13] P. Kroll, H. Moutarde, F. Sabatié, Eur. Phys. J. C 73 (2013) 2278 [1210.6975].

[14] A. Airapetian et al., JHEP 0114 (2012) 077 [1310.5081]. 